\title{
Poliarterite Nodosa Localizada em Membros Inferiores Simulando Tumoração de Panturrilhas
}

\section{Localized Polyarteritis Nodosa Resembling Tumoral Masses on Lower Extremities}

\author{
Mauro Goldfarb $^{(1)}$, Joaquim Jaguaribe Nava Ribeiro(2), Swami José Guimarães ${ }^{(3)}$
}

\section{RESUMO}

É apresentado um caso inusitado de poliarterite nodosa em uma mulher de 57 anos, que se manifestou por aumento progressivo das panturrilhas, com discreta manifestação sintomática local. Entretanto, o achado resultou numa investigação laboratorial e imagenológica, mas que só teve o diagnóstico esclarecido após a realização de estudo histopatológico de biópsia muscular da panturrilha, que demonstrou tratar-se de caso de poliarterite nodosa localizada, sem nenhuma manifestação sistêmica. Revisão da literatura é apresentada.

Palavras-chave: poliarterite nodosa, vasculite, músculos gastrocnêmicos.

\section{INTRODUÇÃO}

A poliarterite nodosa (PAN) foi inicialmente descrita em 1886 por Kussmaul e Meier, que apresentaram os aspectos anatomopatológicos de um caso fatal de um adolescente de 17 anos de idade ${ }^{(1)}$. Desde essa época, sempre foi considerada uma doença sistêmica grave e de mau prognóstico, determinando infartos e isquemias resultantes do comprometimento inflamatório de vasos de pequeno e médio calibre em múltiplos órgãos. Somente no século seguinte, na década de 1950, com a introdução da corticoterapia e, mais ainda, após a associação desse tratamento com os imunossupressores, como a ciclofosfamida, surgiu uma expectativa mais otimista quanto a sua morbimortalidade ${ }^{(2)}$. Mais recentemente, o melhor conhecimento da diversidade clínica da PAN permite utilizar tratamentos mais agressivos apenas nas formas de pior prognóstico, com acometimento do tubo digestivo ou cardíaco, por exemplo ${ }^{(3)}$. Na década de 1970, formas localizadas da doença começaram a ser

\begin{abstract}
An unusual case of polyarteritis nodosa is presented in a 57 year-old woman. Progressive enlargement of her calf muscles with no significant complaints was the clinical presentation. Laboratorial and radiological investigations were not diagnostic. Unexpectedly, the histopathological study was compatible with localized polyarteritis nodosa of her calfs. Literature review is presented.
\end{abstract}

Keywords: polyarteritis nodosa, vasculitis, calf muscles.

descritas ${ }^{(4)}$. Denominada poliarterite nodosa cutânea localizada, de evolução benigna e bom prognóstico, acometia apenas a pele, o sistema musculoesquelético e, por vezes, $\mathrm{o}$ sistema nervoso periférico. A partir daí, descrições de casos envolvendo uma única víscera, mais freqüentemente mama e órgãos geniturinários, têm sido publicadas. Os autores relatam um caso de PAN com comprometimento isolado de ambos os músculos gastrocnêmicos, achado raramente descrito na literatura.

\section{CASO CLÍNICO}

AO, sexo feminino, 57 anos, parda, casada, do lar, natural do Rio de Janeiro. Há cerca de quatro anos, dor, parestesias e aumento insidioso do volume das panturrilhas, que se tornaram endurecidas. As dores eram exacerbadas com a deambulação. Paciente tabagista de um maço/dia e etilista social. Em tratamento para diabetes melito tipo II, além de hipertensão arterial controlada com dieta hipoglicídica e

Recebido em 16/10/06. Aprovado, após revisão, em 08/03/07

Hospital dos Servidores do Estado (HSE) - Ministério da Saúde - Rio de Janeiro.

1. Assistente do Setor de Reumatologia do HSE e professor adjunto e coordenador do Internato de Clínica Médica da Faculdade de Medicina da Universidade Estácio de Sá (Unesa).

2. Assistente do Setor de Reumatologia do HSE.

3. Chefe do Setor de Reumatologia do HSE

Endereço para correspondência: Mauro Goldfarb, Rua Mascarenhas de Morais, 225/801, CEP 22030-040, Copacabana, Rio de Janeiro, RJ, Brasil,

e-mail: m.gold@terra.com.br 
hipossódica, glibenclamida, nifedipina, enalapril e digoxina. Ao exame, foi observado comprometimento importante dos músculos gastrocnêmicos. O direito media $60 \times 71 \times$ $26 \mathrm{~cm}$ e o esquerdo, $58 \times 52 \times 25 \mathrm{~cm}$.

Investigação complementar: hemograma com 5.080.000 hem $/ \mathrm{mm}^{3}$; Ht: 42,7\%; Hb: 13,8 g\%; leucograma com 15.900

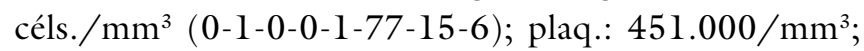
VSH: $48 \mathrm{~mm}$; glicose $100 \mathrm{mg} \%$; Hb glicosilada $7,1 \%$; uréia $23 \mathrm{mg} \%$; creatinina $0,8 \mathrm{mg} \%$; colesterol total 270 mg\%; Anca: não-reativo; anticardiolipina IgG 11 GPL; IgM 1,6 MPL. Eletroneuromiografia dos membros inferiores revelou "polineuropatia sensitivo-motora". EcoDoppler venoso e arterial dos Msls: "discreta ectasia de veias tributárias da safena interna esquerda (segmento abaixo do trocanter interno). Veia safena externa esquerda apresenta espessamento de sua íntima. Insuficiência de grau discreto na veia femural comum direita". Color Doppler arterial do membro inferior esquerdo e direito com fluxos normais. Exame realizado com mapeamento do fluxo em cores. Ressonância nuclear magnética (RNM) de panturrilhas: "ausência de lesão óssea. Áreas heterogêneas permeativas, de sinal hiperintenso em T2, DP e STIR e iso a levemente hiperintenso em Tl, situadas no interstício dos músculos peroneiro breve e longo da perna $\mathrm{E}$ e tibial anterior e soleus da perna $\mathrm{D}$, de natureza inespecífica, devendo corresponder a edema hemorrágico por contusão/estiramento muscular. Demais músculos com morfologia e sinal normais. Feixes vasculoneurais anatômicos. Tecido celular subcutâneo sem alterações" (Figuras l e 2).

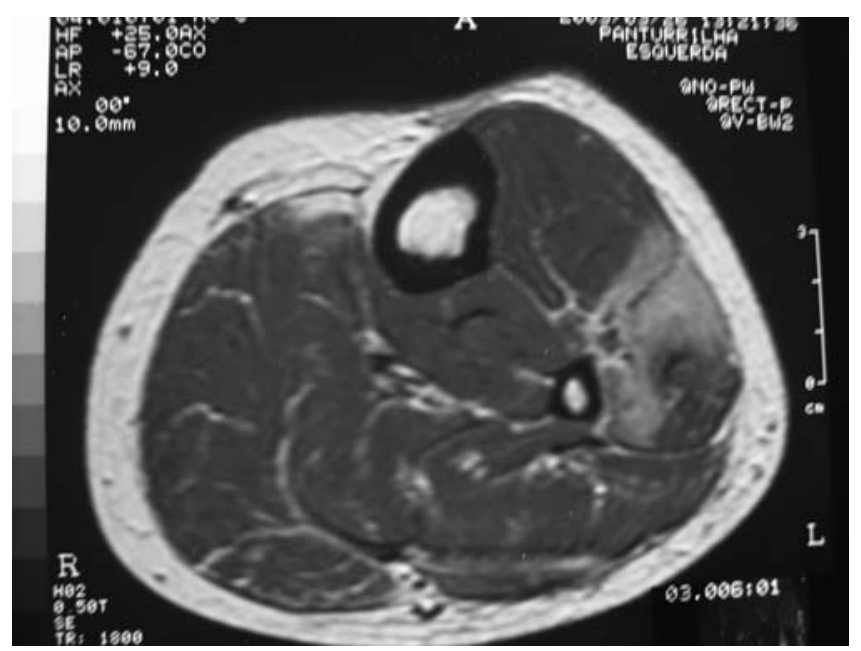

Figura 1 - Áreas heterogêneas permeativas, de sinal iso a levemente hiperintenso em T1, situadas no interstício dos músculos peroneiro breve e longo da perna E e tibial anterior e soleus da perna D.

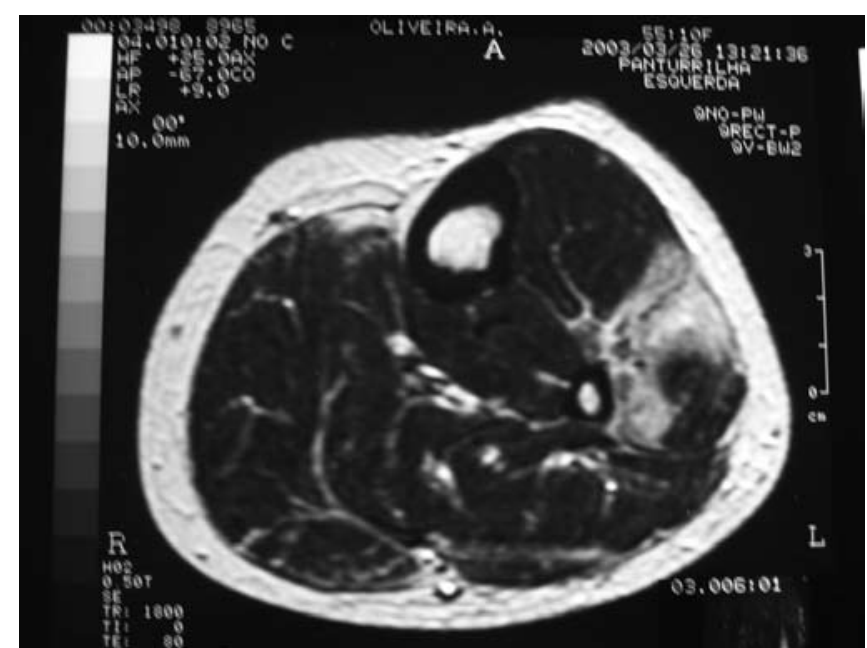

Figura 2 - Áreas heterogêneas permeativas, de sinal hiperintenso em T2, DP e STIR.

Biópsia muscular do gastrocnêmico direito revelou "miosite associada à vasculite de pequeno e médio calibre, com trombose, recanalização e lesão aguda da parede do vaso (necrose fibrinóide)" (Figuras 3 e 4 ).

Iniciado prednisona $20 \mathrm{mg} /$ dia, com melhora progressiva da dor e do aumento de volume das panturrilhas.

\section{DISCUSSÃO}

De acordo com a classificação de Chapell Hill, elaborada em 1992, a mais recente e mais utilizada, que reúne esse heterogêneo grupo de doenças, a PAN foi colocada no grupo das vasculites de médios vasos, não sendo mais aceita a presença de processo inflamatório abrangendo os vasos de pequeno calibre, como as arteríolas, capilares e vênulas. Entretanto, esse conceito foi estabelecido para

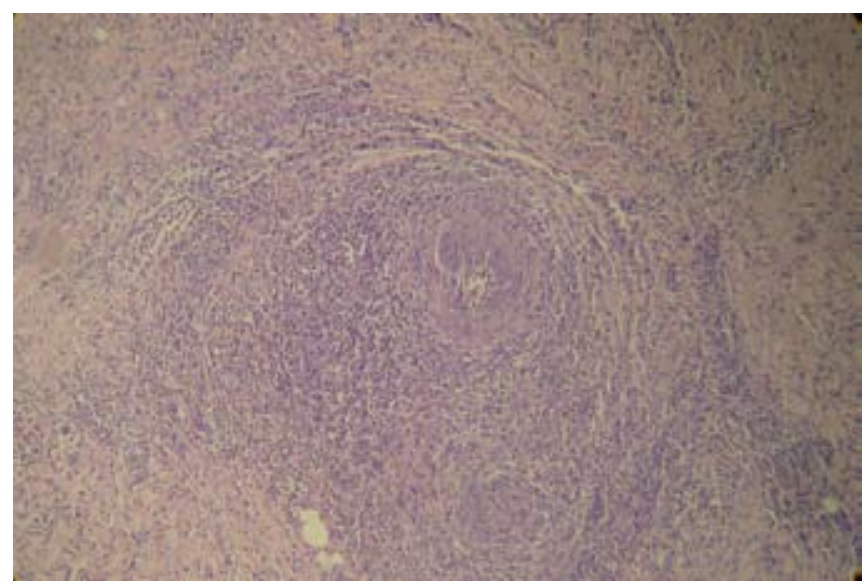

Figura 3 - Trombose vascular, infiltrado polimorfonuclear permeando a parede vascular e necrose fibrinóide focal da parede. 


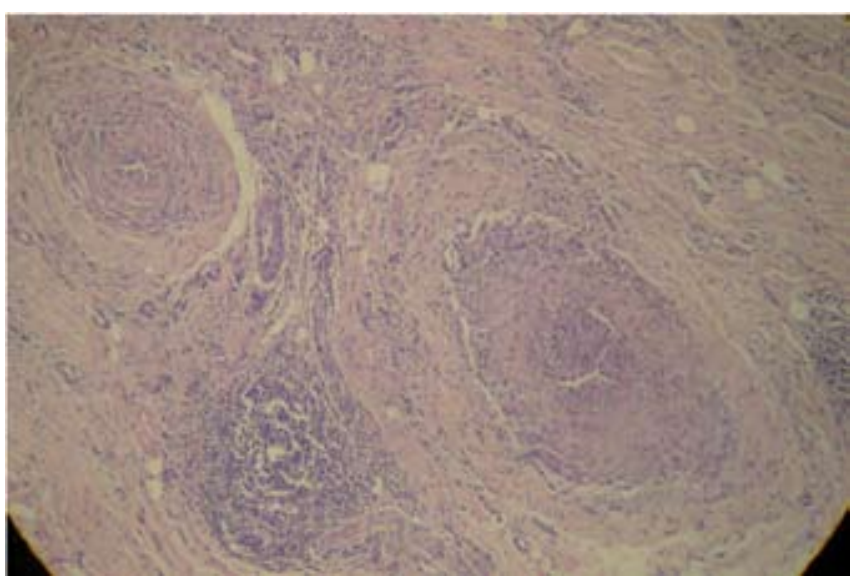

Figura 4 - Dois vasos exibem hiperplasia intimal segmentar, fibrose da média e infiltrado mononuclear na adventícia.

a forma sistêmica da doença ${ }^{(5)}$. As formas localizadas não são citadas nessa classificação. São consideradas formas localizadas as que acometem um órgão isolado, sem as manifestações sistêmicas observadas na PAN, ou quando apenas pele, tecido subcutâneo, nervos periféricos ou aparelho musculoesquelético são comprometidos ${ }^{(3)}$.

O caso descrito constitui forma rara de apresentação da PAN, comprometendo apenas o tecido muscular das panturrilhas. Autores nacionais já descreveram essa apresentação em uma criança, mas foi publicada na literatura estrangeira ${ }^{(4)}$.

\section{REFERÊNCIAS}

1. Kussmaul A, Maier K: Über eine bischer nicht beschreibene eigenthümliche Arterienerkrankung (Periarteritis nodosa), die mit Morbus Brighitii und rapid fortschreitender allgemeiner Muskellähmung einhergeht. Dtsch Arch Klin Med 1:484, 1866.

2. Fauci AS, Haynes BF, Katz P: The spectrum of vasculitis: clinical, pathologic, immunologic and therapeutic considerations. Ann Intern Med 89: 660, 1978.

3. Diaz-Perez J, Winkelman RK: Cutaneous polyarteritis nodosa. Arch Dermatol 110: 407-14, 1974.

4. Pereira BAF, Silva NA, Ximenes AC, Alvarenga SL, Barbosa VS: Cutaneous polyarteritis nodosa in a child with positive antiphospholipid and p-Anca. Scand J Rheumatol 24: 386-8, 1995.

5. Jennete JC, Falk RP, Andrassy K, et al: Nomenclature of systemic vasculitis. Proposal of an international consensus conference. Arthritis Reum 37: 187-92, 1994.
Os casos relatados incluíram tanto pacientes pediátricos como casos na décima década de vida. Poucos são os casos descritos na literatura. O início pode ser agudo, com dor e rápido aumento de volume da musculatura ${ }^{(6)}$, ou insidioso ${ }^{(7)}$ como no nosso caso. Febre pode estar presente ${ }^{(8)}$. O aumento progressivo da lesão pode sugerir uma tumoração( ${ }^{(9)}$. Outras localizações, como o antebraço, além da panturrilha, podem ser encontradas. Apesar de achados laboratoriais terem sido eventualmente descritos, como FAN, Anca ou anticorpos anticardiolipina $^{(4,10)}$, não são auxiliares no diagnóstico, nem há evidências de doenças sistêmicas associadas. $\mathrm{O}$ diagnóstico é esclarecido ao ser realizada a biópsia da tumoração, geralmente sem que a possibilidade de uma vasculite tivesse sido suspeitada, uma vez que não há outros sinais sistêmicos da doença. O diagnóstico diferencial inclui tumorações benignas e malignas musculares, doenças infecciosas de baixa virulência, como tuberculose, infartos musculares em dializados ou diabéticos, rupturas musculares e aneurismas. O tratamento é eficaz e, utilizando-se corticosteróides em dose imunossupressora, ocorre redução progressiva da tumoração ${ }^{(11,12)}$. Constitui-se, portanto, em condição benigna, de bom prognóstico, mas que deve ser conhecida para incluí-la no diagnóstico diferencial das tumorações musculares.

\section{Declaramos a inexistência de conflitos de interesse.}

6. Garcia F, Pedrol E, Casademont J, et al: Polyarteritis nodosa confined to calf muscles. J Rheumatol 19: 303-5, 1992.

7. Esteva-Lorenzo FJ, Ferreiro JL, Tardaguila F, et al: Pseudotumor of the muscle associated with necrotizing vasculitis of mediumand small-sized arteries and chronic myositis. Skeletal Radiol 23(7): 572-6, 1994.

8. Ferrairo JE, Saldane MJ, Azevedo SJ: Polyarteritis manifesting as calf myositis and fever. Am J Med 80: 312-5, 1986.

9. Hiar I, Ansart S, Volant A, Leroy JP, Pennec YL: Periartérite noueuse musculaire localisée pseudotumorale. Rev Med Interne 23: 792-4, 2002.

10. Astidillo LM, Rigal F, Couret B, Arlet-Suau E: Localized presentation of polyarteritis nodosa. J Rheumatol 28: 2758-9, 2001.

11. Hall Charles, Mongey A-B: Unusual presentation of polyarteritis nodosa. J Rheumatol 28(4): 871-3, 2001.

12. Laitinen $\mathrm{O}$, Haltia M, Landevirta J: Polyarteritis confined to lower extremities. Scand J Rheumatol 11: 71-4, 1982. 